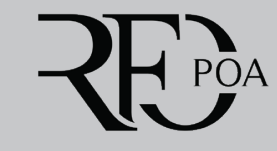

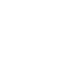

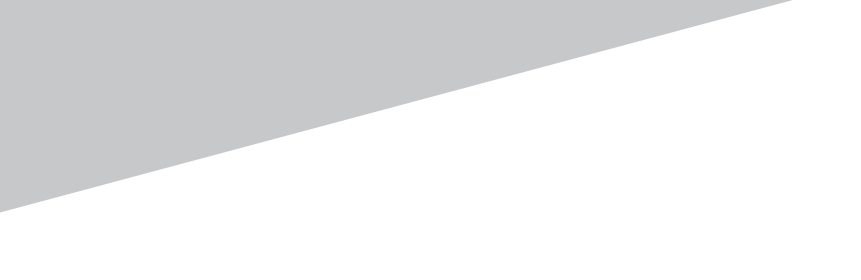

\title{
AvALIAÇÃO E MANEJO DA ANSIEDADE E FOBIA ODONTOLÓGICA: A PSICOLOGIA NA FORMAÇÃO DO CIRURGIÃO-DENTISTA
}

\author{
Evaluation and management of anxiety and dental fear: \\ psychology in the formation of the dentist
}

(DGuilherme Borsato Gomes ${ }^{a}$, (DCecília Luiz Pereira Stabileb, (DVanessa Santiago Ximenes ${ }^{c}$

\section{RESUMO}

O medo e a ansiedade odontológica surgem por diversos fatores, entre eles, a exposição à experiências traumáticas anteriores com envolvimento de agulhas, injeções e instrumentos que possam causar incômodo. Os pacientes ansiosos tendem a adiar consultas, evitam os tratamentos odontológicos e só se dispõe às visitas ao dentista quando aparecem sintomas dolorosos, logo o tratamento tardio tende a ser mais invasivo causando ainda mais prejuízo emocional ao paciente, consequente agravo a tal sentimento e, por sua vez, origina um ciclo vicioso no qual evitar o tratamento agrava ainda mais a condição de saúde bucal. Cabe aos profissionais da Odontologia identificar a ansiedade nos pacientes e fazer uso de recursos de manejo dessa ansiedade de modo a possibilitar um tratamento odontológico eficaz e com menor prejuízo emocional ao paciente. Com este estudo pretende-se avaliar por meio de uma revisão integrativa de literatura, a importância do estudo da Psicologia pelo profissional da Odontologia e expor algumas técnicas de enfrentamento da ansiedade odontológica, com base em evidências científicas.

Palavras-chave: Odontologia. Psicologia. Assistência odontológica. Ansiedade. Manobra psicológica.

\begin{abstract}
Dental fear and anxiety arise from several factors, among them, exposure to previous traumatic experiences with needles, injections and instruments that can cause discomfort. Anxious patients tend to reschedule appointments, avoid treatments and are only available for visits at the dentist when painful symptoms appear, so delayed treatment tends to be more invasive and cause even more emotional damage to the patient, consequently aggravate this feeling and, in turn, create a vicious cycle in which avoiding treatment only makes the oral health condition even worse. It is up to professionals of Dentistry to identify anxiety in patients and make use of resources to manage this anxiety in order to enable an effective dental treatment with less emotional damage to the patient. The aim of this study is to evaluate, by means of a integrative literature review, the importance of the study of Psychology by the dental professional and to expose the techniques for coping with dental anxiety, based on scientific evidences.
\end{abstract}

Keywords: Dentistry. Psychology. Dental care. Anxiety. Handling, psychological.

\footnotetext{
${ }^{a}$ Graduação em Odontologia, Universidade Estadual de Londrina, Londrina, PR, Brasil.

${ }^{\text {b }}$ Departamento de Medicina Oral e Odontologia Infantil, Universidade Estadual de Londrina, Londrina, PR, Brasil.

${ }^{\mathrm{c}}$ Departamento de Psicologia Geral e Análise do Comportamento, Universidade Estadual de Londrina, Londrina, PR, Brasil.
}

Autor de correspondência: Guilherme Borsato Gomes - E-mail: guilhermeborsato7@gmail.com

Data de envio: 12/03/2020 | Data de aceite: 13/05/2020 


\section{INTRODUÇÃO}

A ansiedade diante do atendimento e tratamento odontológico interfere diretamente nos cuidados em saúde bucal ${ }^{1}$. Diante de um quadro de ansiedade, diminuem as chances do paciente tanto ir às consultas quanto colaborar com o dentista, fatos que dificultam o trabalho do profissional da Odontologia, além de trazer prejuízos para a saúde bucal e integral do paciente.

Em linhas gerais, a ansiedade é caracterizada por um sentimento de temor e apreensão, levando a uma tensão e medo derivados da antecipação de perigo ou algo desconhecido. Já o medo passa a ser caracterizado como transtorno psicológico quando é expressado pelo paciente de forma desproporcional ao estímulo, ou de tal modo que é considerado fora do normal, e interfere na realização de tarefas rotineiras, pois o paciente tende a fugir ou se esquivar não apenas do que lhe faz sentir medo, mas também de estímulos que se assemelham funcionalmente àquele gerador de sofrimento, impactando sua saúde emocional e qualidade de vida ${ }^{2}$.

Como os indivíduos com transtornos de ansiedade em geral superestimam o perigo nas situações que temem ou evitam, a determinação primária do quanto o medo ou a ansiedade são excessivos ou fora de proporção é feita pelo clínico, levando em conta fatores contextuais culturais. Muitos dos transtornos de ansiedade se desenvolvem na infância e tendem a persistir se não forem tratados ${ }^{3}$.

Transtornos fóbicos específicos consistem em medos persistentes, irracionais e intensos de situações, circunstâncias ou objetos específicos. Uma fobia específica consiste em medo e ansiedade diante de uma situação ou um objeto em particular. A situação ou o objeto é geralmente evitado quando possível; mas, caso a exposição ocorra, a ansiedade tende a estar presente em sequência ${ }^{3}$. Possíveis origens da ansiedade relacionada ao atendimento odontológico encontram-se em práticas culturais, crenças e ensinamentos advindos da própria sociedade, de tal forma é-nos ensinado que a felicidade é algo cotidiano e almejado, enquanto o medo, a dor e a tristeza devem ser evitados, como se tais sentimentos e acontecimentos não fizessem parte da história e vivência como seres humanos e por isso devessem ser reprimidos ou eliminados. Partindo do princípio que os estímulos (como objetos ou situações) não tem um valor em si, ou seja, não são reforçadores ou aversivos em si mesmos, e sim, adquirem tais funções ao longo da história de vida do indivíduo, deve-se também considerar tal história, como forma de entender como determinados estímulos (como um jaleco branco, por exemplo) podem ter adquiro um significado aversivo para determinada pessoa 4 .

De modo geral, o medo e a ansiedade ao dentista parecem ser multifacetados, de modo que o indivíduo não apenas evita a ida às consultas, mas também tende a ter saúde bucal deficiente, visto que, evitando a situação ansiogênica, o paciente se submete a um descuido maior a respeito de sua responsabilidade com os cuidados bucais, levando a necessidade de procedimentos mais invasivos - consequentemente mais estressantes - quando finalmente procura o cirurgião-dentista $(\mathrm{CD})^{5}$.

Embora a literatura que aborda questões psicológicas em pacientes adultos, vinculadas ao medo de dentista, seja escassa no Brasil, compreender os aspectos psicológicos que interferem no tratamento dentário é essencial na prevenção e tratamento da ansiedade, medo e fobias do atendimento odontológico ${ }^{5}$.

Sendo assim, este estudo almeja (a) identificar variáveis relacionadas a aversão ao cenário de tratamento odontológico, e (b) analisar técnicas e recursos de enfrentamento de ansiedade, medo e fobia no contexto odontológico descritas na literatura. Para tanto, este estudo utiliza-se de artigos que apresentam o tema "Psicologia aplicada à Odontologia", além de textos recentes que embasam a evidenciação da importância de pesquisas e mais estudos acerca dos aspectos psicológicos do paciente, sendo isso de interesse do CD e também de estudantes em formação na Odontologia. 


\section{MATERIAIS E MÉTOdOS}

O estudo foi realizado por meio de uma revisão integrativa da literatura que objetivou analisar a ansiedade e o medo odontológico em pacientes adultos. A coleta de bibliografia foi realizada a partir da busca de informações online na Biblioteca Virtual de Saúde (BVS

- bvsalud.org), nas bases de dados LILACS e Google Scholar.

Primeiramente, foram pesquisadas publicações que continham no título ou no resumo o descritor "Ansiedade odontológica". Na sequência, a busca foi refinada para (a) artigos, teses e dissertações com texto completo disponível gratuitamente para download, (b) nos idiomas português e inglês, (c) contendo no assunto principal o tema "Ansiedade em Contexto Odontológico", (d) entre os anos de 1990 a 2019 e (e) artigos, teses e dissertações que descreveram pesquisas com adultos ou estudos de revisão de literatura. Os critérios de exclusão foram: artigos, teses e dissertações que não contemplavam em seu título ou resumo os descritores apontados, textos incompletos e/ou indisponíveis para download e pesquisas realizadas com crianças e adolescentes. A partir dos critérios de inclusão e exclusão, os artigos selecionados passaram por uma revisão por dois juízes independentes, formalizando então o material de análise do presente estudo. Em seguida realizou-se a descrição dos dados do escopo selecionado e a determinação de pontos específicos para a discussão.

O segundo ponto avaliado foi a presença da Psicologia na formação acadêmica do CD no Brasil. A obtenção de dados que declaram a presença ou não de matérias que contemplam conteúdos da Psicologia nas universidades, foi realizado por meio das plataformas digitais das instituições, nas quais foram coletadas as informações das Matrizes Curriculares de cada Universidade Pública Brasileira que fornece o curso de Odontologia, e em seguida foi realizada a descrição dos dados obtidos.

\section{Resultados}

Foram identificados 19 artigos e 1 dissertação, que preencheram os critérios de inclusão estabelecidos (Figura 1). Na sequência serão descritos os seguintes tópicos referentes ao escopo do presente estudo: conceitos e prevalência de ansiedade, medo e fobia no contexto odontológico, a Psicologia na formação do CD no Brasil, métodos de avaliação da ansiedade em contexto odontológico e técnicas de enfrentamento da ansiedade, medo e fobia (Quadro 1). As técnicas de enfrentamento da ansiedade foram complementadas com materiais específicos da Psicologia. 

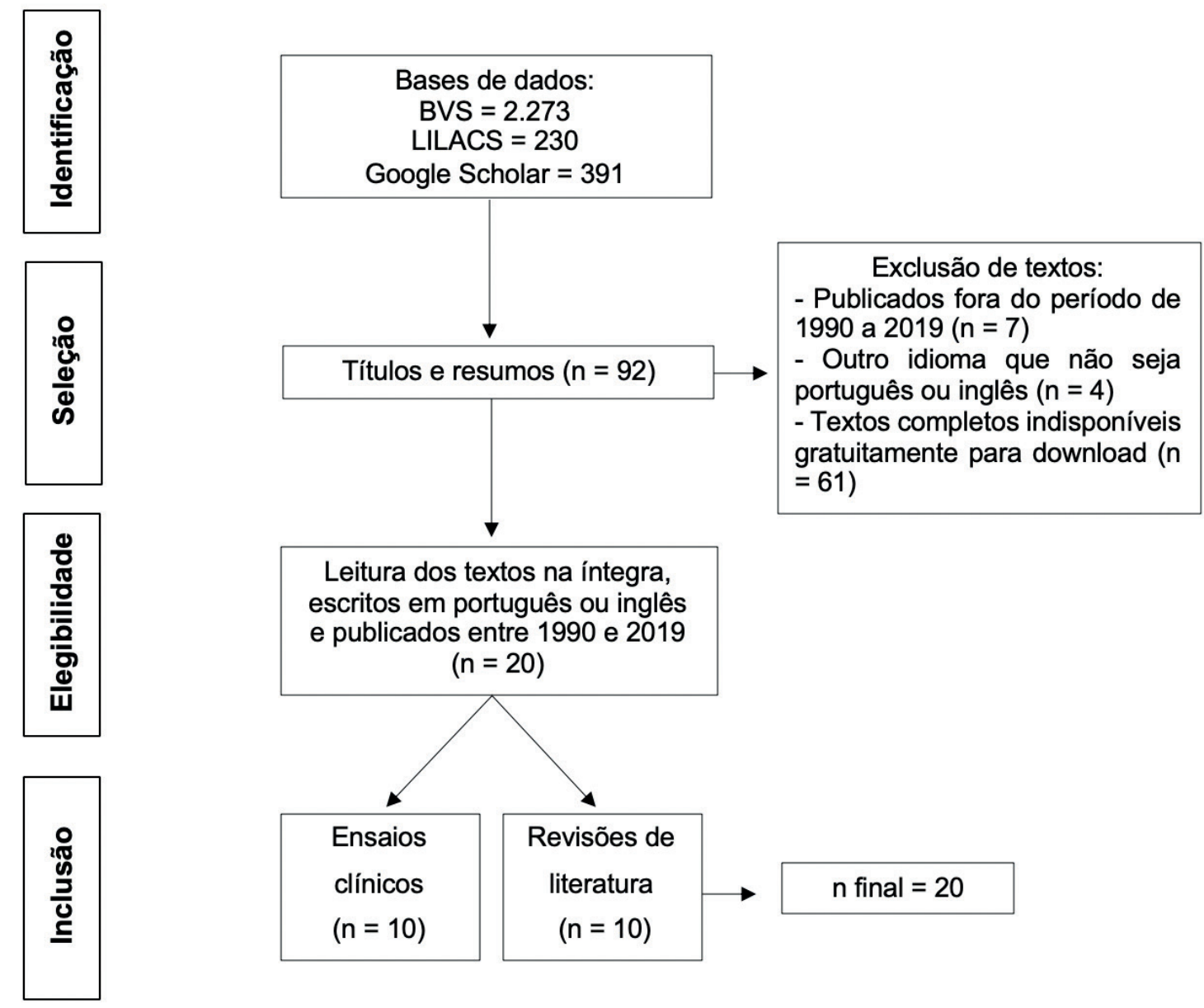

Figura 1: Diagrama de fluxo da seleção dos estudos.

Quadro 1: Principais informações de cada material selecionado.

\begin{tabular}{|l|l|}
\hline Estudo & Principais achados \\
\hline Ackley & \\
\hline $\begin{array}{l}\text { Armfield e } \\
\text { Heaton }\end{array}$ & $\begin{array}{l}\text { A respiração lenta mostra-se eficaz na redução da frequência cardíaca de pacientes } \\
\text { que se queixam de ansiedade }\end{array}$ \\
\hline Bernson et al. ${ }^{8}$ & $\begin{array}{l}\text { A comunicação paciente-dentista é essencial para que o tratamento prossiga } \\
\text { sem problemas }\end{array}$ \\
\hline Carter et al. ${ }^{5}$ & $\begin{array}{l}\text { Devido à abstenção às consultas o paciente se submete a um descuido maior } \\
\text { com os cuidados bucais, levando-o a necessitar de procedimentos mais invasivos }\end{array}$ \\
\hline Carvalho et al. ${ }^{9}$ & $\begin{array}{l}\text { Falta de recursos econômicos, o descaso com a saúde bucal, o gênero e a idade } \\
\text { podem aumentar o grau de ansiedade }\end{array}$ \\
\hline Dailey et al. ${ }^{10}$ & $\begin{array}{l}\text { O manejo da ansiedade deve ser individualizado a cada paciente, abordando suas } \\
\text { necessidades e características pessoais }\end{array}$ \\
\hline Hakeberg et al. ${ }^{11}$ & $\begin{array}{l}\text { A aplicação de métodos não farmacológicos para manejo da ansiedade odonto- } \\
\text { lógica mostraram-se duradouros }\end{array}$ \\
\hline
\end{tabular}




\begin{tabular}{|c|c|}
\hline Estudo & Principais achados \\
\hline Kim et al. ${ }^{12}$ & Pacientes tratados com música relataram menos ansiedade durante a cirurgia \\
\hline Klassen et al. ${ }^{13}$ & $\begin{array}{l}\text { Música é eficaz na redução da ansiedade e dor em pacientes submetidos a proce- } \\
\text { dimentos odontológicos }\end{array}$ \\
\hline $\begin{array}{l}\text { Klingberg e } \\
\text { Broberg }^{14}\end{array}$ & $\begin{array}{l}\text { A prevalência de ansiedade por fatores odontológicos foi estimada em 9\%, com } \\
\text { uma diminuição na prevalência de acordo com a idade }\end{array}$ \\
\hline Lopes et al. ${ }^{15}$ & $\begin{array}{l}\text { O teste psicométrico de ansiedade dentária é valido para sua utilização como } \\
\text { forma de diagnóstico e nivelamento da ansiedade do paciente }\end{array}$ \\
\hline Mayer et al. ${ }^{4}$ & $\begin{array}{l}\text { Pacientes que já apresentam ansiedade em contexto odontológico, quanto maior o } \\
\text { tempo desde a última consulta, maior será o grau de ansiedade em uma próxima visita }\end{array}$ \\
\hline $\begin{array}{l}\text { Milgrom e } \\
\text { Weinstein }^{16}\end{array}$ & $\begin{array}{l}\text { Os medos adquiridos na infância por experiência direta com tratamento doloroso ou } \\
\text { indiretamente, podem persistir na idade adulta e prejudicar o tratamento odontológico }\end{array}$ \\
\hline $\begin{array}{l}\text { Murrer e Fran- } \\
\text { cisco }^{17}\end{array}$ & $\begin{array}{l}\text { O estudo da psicologia aplicada à odontologia mostra-se eficaz no manejo da } \\
\text { ansiedade odontológica e prevenção do agravamento dos medos }\end{array}$ \\
\hline Nathan $^{18}$ & $\begin{array}{l}\text { A convicção de que o paciente será submetido a algum tipo de dor durante o } \\
\text { atendimento dificulta a continuidade do tratamento proposto }\end{array}$ \\
\hline Pereira et al. ${ }^{19}$ & $\begin{array}{l}\text { A prevalência de ansiedade não mostrou-se de forma significativa nas variáveis } \\
\text { pesquisadas: gênero, idade, renda, nível de instrução, sendo um caractere } \\
\text { individual de cada paciente }\end{array}$ \\
\hline Peronio et al. ${ }^{20}$ & $\begin{array}{l}\text { As manifestações relacionadas ao medo de dentista têm como consequência a } \\
\text { abstenção às consultas e a piora da condição bucal }\end{array}$ \\
\hline Silva ${ }^{21}$ & $\begin{array}{l}\text { Experiência anterior de trauma em contexto odontológico é um fator determi- } \\
\text { nante sobre o grau de ansiedade do paciente }\end{array}$ \\
\hline Skaret et al. ${ }^{22}$ & $\begin{array}{l}\text { A ansiedade dental é uma das principais razões para a alta frequência } \\
\text { de consultas perdidas }\end{array}$ \\
\hline Ulhoa et al. ${ }^{23}$ & $\begin{array}{l}\text { A formação do CD deve ser complementada com estudos da psicologia como } \\
\text { forma de auxiliar o profissional a lidar de forma mais efetiva com os aspectos } \\
\text { psicossociais dos pacientes }\end{array}$ \\
\hline
\end{tabular}

Com base no material selecionado sobre as matrizes curriculares das Universidades Públicas no Brasil, observou-se que das 53 universidades com o curso de graduação em Odontologia, 44 delas apresentam em seu projeto pedagógico alguma disciplina de Psicologia. A título de ilustração, na Universidade Federal de Juiz de Fora há a disciplina Psicologia Aplicada à Odontologia, a qual busca assegurar aos acadêmicos oportunidades de: adquirir conhecimentos sobre a Psicologia Humana e os diversos tipos de comportamentos, individualizando cada paciente; aprender a lidar com pacientes nas diversas áreas da Odontologia, correspondendo aos seus anseios e motivando-os como unidades biopsicossociais; conhecer as formas de condicionar e modelar o comportamento dos pacientes. Entretanto, observa-se que essas disciplinas, em sua maior parcela, são de oferta semestral nas grades curriculares, estão dissolvidas em outras disciplinas, apresentam carga horária insuficiente (média de 30 horas) e, consequentemente, tendem a não aprofundar os conhecimentos da Psicologia dado o pouco tempo disponibilizado para a inserção da Psicologia nas grades curriculares. 


\section{Discussão}

\section{Conceitos e prevalência de ansiedade, medo e fobia no contexto odontológico}

Klingberg e Broberg ${ }^{14}$ investigaram a literatura publicada entre 1982 a 2006, e estimaram a prevalência de ansiedade a fatores odontológicos no mundo em $9 \%$. Estudos realizados nos Estados Unidos, Dinamarca e Suécia demonstraram predominância de ansiedade e fobia ao atendimento odontológico em 10\%, 10,2\% e 6,7\% da população, respectivamente. Nos países em desenvolvimento há pouca pesquisa e investigações científicas sobre o assunto, ao passo que, no Brasil, a literatura em questão, tomando como base pacientes adultos e jovens adultos, apresenta-se escassa ${ }^{17}$ ( 9 estudos encontrados).

Embora os estudos sobre controle da dor tenham evoluído em todo o mundo, Skaret et al. ${ }^{22}$ refere que o medo extremo e irracional frente ao tratamento odontológico ocorre entre 5 e $10 \%$ da população adulta, a qual, por sua vez, tende a evitar parcial ou completamente o tratamento, por aversão à potencial sensação dolorosa, tendo como consequência uma saúde oral e qualidade de vida deficientes.

A expressão "medo", na literatura, encontra-se definida em uma diversidade de conjuntos e proposições. Portanto, tomando como base resoluções do estudo da Psicologia, pode-se considerar "medo" como um evento amplo, exposto em inúmeros contextos culturais e que se mostra presente em todas as camadas sociais; é uma das emoções humanas que tem como papel fundamental nos alertar sobre o perigo ${ }^{17}$.

O indivíduo tende a evitar perigos e ameaças em potencial, pois estes podem comprometer sua integridade e bem-estar ${ }^{24}$. Portanto, o medo pode ser considerado uma reação de sobrevivência e preservação através de respostas de luta ou fuga de estímulos aversivos que podem colocar em risco a integridade do indivíduo ${ }^{24}$.

A expressão do medo pode apresentar-se no corpo do paciente por meio do aumento significativo da frequência cardíaca e respiratória, sensação de sufocamento, boca seca, sudorese e tremore ${ }^{17,19}$. Por conseguinte, pode ocorrer também maior liberação de secreção gástrica e aumento da motilidade do trato gastrointestinal, levando a uma sensação de mal-estar físico intenso. Além disso, o medo é caracterizado também por uma diminuição do limiar de percepção da dor, proporcionando um aumento da secreção de adrenalina, e consequentemente, ativação dos nociceptores, implicando em maior sensibilidade aos estímulos físicos ${ }^{9}$. Tais reações fisiológicas são ainda mais evidentes em pacientes sistemicamente comprometidos (pacientes hipertensos, cardiopatas, com doenças respiratórias) ${ }^{9}$.

Murrer e Francisco ${ }^{17}$ considerando o ponto de vista psicológico do paciente descrevem que a manifestação do medo no indivíduo leva a diversas consequências, as quais também devem ser consideradas pelo CD no atendimento odontológico. Ao sentir-se com medo, o paciente tende a apresentar menor tolerância à frustração, menor chance de emitir comportamentos da classe das habilidades sociais, dificuldade de concentração (principalmente na tentativa do odontólogo de explicar e orientar sobre os procedimentos a serem realizados), e maior chance de sentir angústia, apreensão, insegurança, mal-estar indefinido e até mesmo, em alguns casos, apresentar crises de choro. Na iminência de um possível episódio aversivo, o paciente tende a evitar ambientes de estimulação e lutar contra esse movimento na busca de sua preservação, sendo por exemplo, mais ríspido com o dentista ${ }^{25}$.

Segundo Nathan ${ }^{18}$ um dos principais fundamentos que parece interferir no comportamento de grande parte dos pacientes que buscam por um atendimento odontológico é a conviç̧ão de que serão submetidos a algum tipo de incômodo ou sensação dolorosa durante o atendimento. Essa crença ou regra, usualmente, inicia-se na infância ou na adolescência e é resultante de experiências dolorosas passadas, desconhecimento dos procedimentos a serem realizados, impossibilidade de ver o que o cirurgião executa durante o procedimento, 
o contato com o ambiente do consultório (cadeira odontológica, instrumentais, motores de alta e baixa rotação), o acesso à informações negativas e depreciativas transmitidas por outras pessoas, sendo esses alguns fatores que podem levar o paciente à esquiva das consultas e consequentemente, no futuro, ir de encontro à intervenções invasivas, causando ainda mais medo, dando origem a um ciclo vicioso no qual fugir do tratamento só agrava ainda mais a condição bucal ${ }^{22}$.

Deste modo, considerando o impacto da ansiedade odontológica e a importância dos conhecimentos técnicos e teóricos, provenientes da Psicologia, é necessário avaliar qual foi a base curricular preconizada na formação do CD no Brasil até a seu encaminhamento para o mercado de trabalho e discutir a importância que tal base influencia nos conhecimentos do profissional quanto ao atendimento do paciente com ansiedade odontológica.

\section{A Psicologia na formação do cirurgião-dentista no Brasil}

A Psicologia aplicada à Odontologia pode ser caracterizada como um conjunto de conhecimentos técnicos e teóricos decorrente da prática da Psicologia clínica, aplicando atividades de avaliação, controle e modificação de comportamentos de indivíduos que fazem um tratamento odontológico ou envolvidos em cenários de cuidados em saúde bucal ${ }^{26}$.

O objetivo desta relação interprofissional entre a Odontologia e a Psicologia é abordar aspectos psicossociais presentes nos métodos de avaliação, tratamento e reabilitação em Odontologia, buscando propiciar e sustentar a saúde geral do indivíduo, bem como prevenir e colaborar eficientemente em situações de tratamento de patologias bucais. Por referir-se a uma área interdisciplinar, são necessárias a colaboração mútua e a incorporação de conhecimentos da Odontologia, Psicologia e das demais ciências da saúde ${ }^{27}$.

Segundo a Organização Mundial de Saúde (OMS), saúde bucal é mais que dentes saudáveis; é a integração da saúde geral e essencial para o bem estar da população ${ }^{5}$. Saúde bucal adequada implica em um indivíduo livre de dor crônica do complexo orofacial, lesões dos tecidos moles bucais e peribucais, defeitos congênitos e outras patologias que afetam os tecidos bucais, dentais e orofaciais ${ }^{21}$. Assim sendo, é necessário conhecer as principais alterações bucais que acometem o paciente, bem como os aspectos psicossociais que influenciam na qualidade de vida deste indivíduo, visto que o paciente deve ser considerado e tratado como um ser biopsicossocial.

A ansiedade, medo e fobia podem ser dificuldades enfrentadas pelo paciente durante a busca por um tratamento odontológico, fato que pode gerar atrasos na continuidade da intervenção, visto que pacientes que se sentem ansiosos tendem a desmarcar consultas, não colaborar com a realização de procedimentos e adiar a visita periódica ao CD ${ }^{21}$. Em 2015, Murrer e Francisco ${ }^{17}$ realizaram um questionário autoaplicável sobre diagnóstico e tratamento da ansiedade odontológica a 967 dentistas e constataram que 35\% deles não aplicam qualquer tipo de avaliação de ansiedade dos pacientes. Os autores afirmaram ainda que os dentistas que fazem avaliação da ansiedade do paciente não usam critérios científicos definidos para mensurar a ansiedade, sendo que essa avaliação é pautada numa conversa informal e pouco estruturada entre paciente e CD. Aproximadamente 32\% dos profissionais participantes relataram utilizar um protocolo específico para a abordagem do paciente ansioso, sendo os mais utilizados: a conversa informal (38\%), a prescrição farmacológica (23\%) ou algum tipo de condicionamento do paciente (17\%); apenas $17 \%$ dos profissionais participantes na pesquisa utilizam de alguma outra abordagem como encaminhamento para Psicólogo ou Médico. Esse achado demonstra uma possível defasagem na aplicação, pelos Cirurgiões-dentistas, dos indícios científicos da área da Psicologia disponíveis sobre a eficácia de diversas formas de avaliação e tratamento da ansiedade. A formação profissional do CD generalista no Brasil situa-se, ainda, muito focada apenas na aquisição de habilidades técnicas e de 
refinamento, assim como o desenvolvimento e sofisticação dos procedimentos técnicos. Há pouco investimento na formação profissional dos cirurgiões-dentistas acerca da identificação e manejo de pacientes com medo do tratamento odontológico, tendo em vista que as Escolas de Odontologia, na maioria das vezes, não contemplam, em seus projetos pedagógicos, uma formação multiprofissional ativa, incluindo principalmente a ciência da Psicologia, nas quais o profissional poderia ser instruído com maior atenção e habilitado para o reconhecimento e correta abordagem do paciente emocionalmente comprometido. Além disso, os profissionais já formados deveriam buscar por formação complementar para aprender a lidar com os aspectos psicossociais inerentes e indissociáveis aos pacientes ${ }^{17,23}$.

A repercussão que a ansiedade odontológica pode ter na vida das pessoas é ampla e dinâmica, não só levando à evasão de cuidados com a saúde bucal, mas também efeitos individuais em geral. A Psicologia na formação do CD, como evidenciado, é de suma importância para que o mesmo seja capaz de acolher o paciente com ansiedade odontológica e realizar a correta abordagem, tendo como base evidências científicas e estudos certificados em relação aos métodos mais aplicáveis na prática clínica odontológica ${ }^{17}$.

\section{Métodos de avaliação da ansiedade em contexto odontológico}

Dailey et $a .^{10}$ evidenciaram que quando o CD sabe o grau de ansiedade odontológica do paciente, essa ansiedade pode se reduzir, provavelmente porque o profissional pode adaptar sua forma de atuar, prestando mais atenção às respostas emocionais do paciente e, além disso, o paciente tende a se tranquilizar, pois o dentista demonstrará um manejo mais adequado dentro da especificidade de cada paciente atendido.

Atualmente, existem diversos métodos para mensurar a ansiedade do paciente, sendo possível agrupá-los em seis grupos: subjetivos (ilustração clínica global embasada em uma entrevista informal), sistemáticos (investigam a ansiedade em suas variadas vertentes utilizando questionários), focais (focam em um aspecto específico da ansiedade do paciente), não-objetivas (testes projetivos), semiobjetivas (utilizam questionários em que os sinais e sintomas específicos são apresentados, bem como as instruções para os quantificar) e objetivas (utilizam de medidas variáveis psicofisiológicas, como cardiovasculares, respiratórias, músculo-esqueléticas $)^{15}$.

Em um estudo publicado por Carvalho et al. ${ }^{9}$, apresenta-se que, dentre as múltiplas estratégias utilizadas para categorizar e mensurar a ansiedade odontológica, existe uma que é mais aplicável e abordada em estudos sobre este tema, que é a Escala de Corah. Tal escala é conhecida como objeto de avaliação das manifestações da ansiedade odontológica desde a década de 1970, sendo amplamente utilizada e traduzida para vários idiomas, pois permite reconhecer objetivamente o nível de ansiedade por meio da soma das respostas fornecidas pelo paciente através das perguntas de múltipla escolha (Quadro 2).

Segundo a escala de Corah, pacientes cuja soma das respostas foi inferior a 5 pontos são considerados muito pouco ansiosos; pontuações entre 6 e 10, são considerados levemente ansiosos; já pacientes que apresentam pontuações entre 11 e 15 são encarados como pacientes moderadamente ansiosos; e somas superiores a 15 pontos, extremamente ansiosos ${ }^{9}$. 
Quadro 2: Perguntas de múltipla escolha da escala de Corah para ansiedade odontológica.

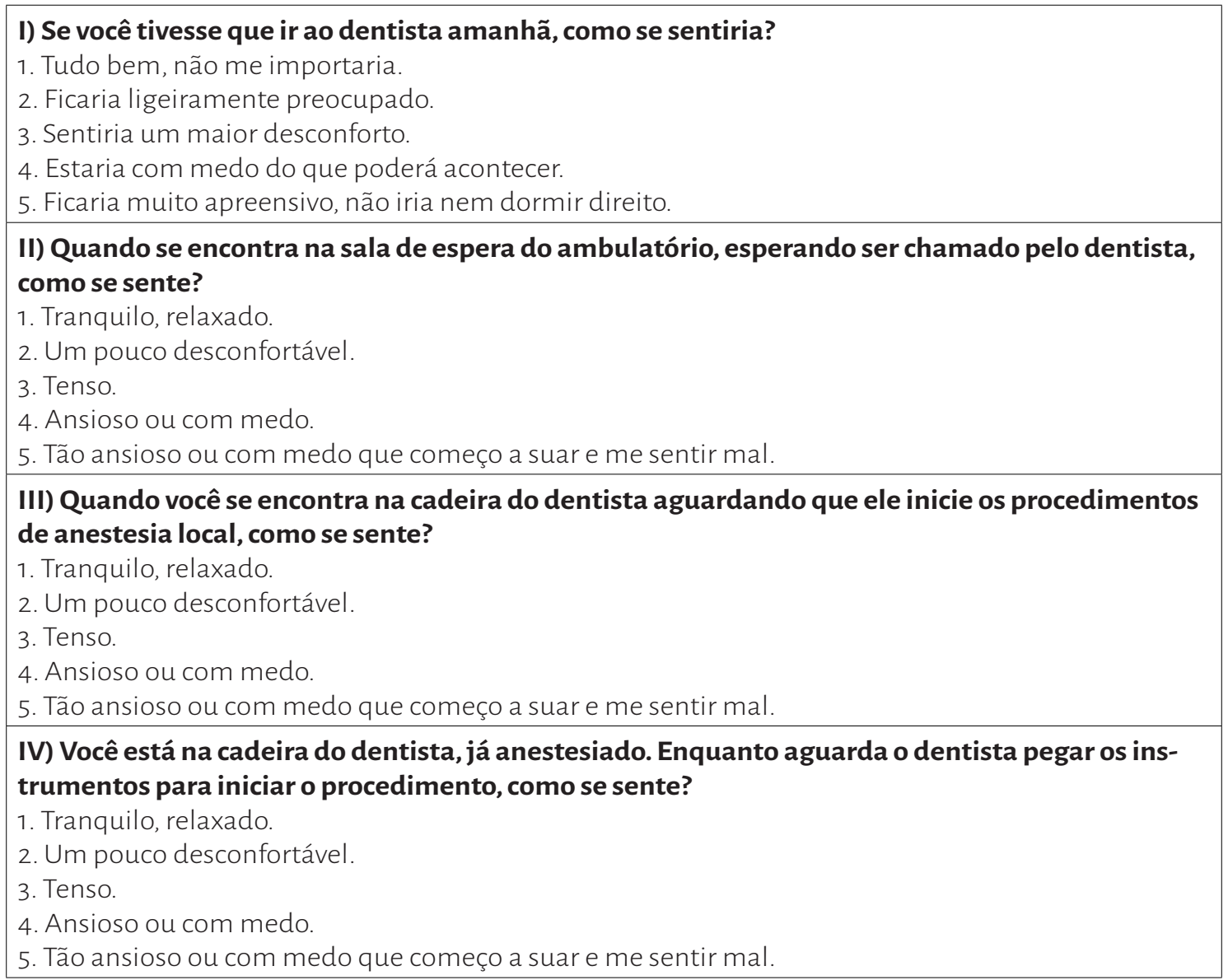

Fonte: Extraído de Hu et al. ${ }^{28}$

Para os profissionais que cuidam da saúde oral, a ansiedade, o medo e fobia são importantes obstáculos na entrega de cuidados a saúde, entretanto, com os instrumentos de avaliação da ansiedade odontológica o profissional torna-se apto a conhecer melhor o perfil emocional do paciente e com isso traçar o melhor plano de tratamento odontológico e, por sua vez, utilizar de técnicas da psicologia para ajudar o paciente a enfrentar a ansiedade e dar sequência ao tratamento, visando um conforto geral ao paciente em ambiente odontológico.

\section{Técnicas de enfrentamento da ansiedade, medo e fobia}

O contexto odontológico pode dispor de muitos eventos aversivos, de modo que o medo de dentista constitua uma queixa frequente em grande parte da população mundial. Os indivíduos com medo de estímulos específicos podem identificar prontamente os aspectos que consideram mais aversivos. Embora os mais comuns desses estímulos sejam injeções, sons, visualização dos instrumentais, a peça de mão e a dor associada ao tratamento odontológico, indivíduos com medo podem identificar qualquer procedimento odontológico ou partes do ambiente do consultório como fator desencadeante de seu medo, justificando a necessidade de se buscar maneiras de manejar a ansiedade e promover um atendimento seguro e de qualidade ao paciente ${ }^{7}$. 


\section{Práticas de manejo da ansiedade para níveis de ansiedade identificados}

Considera-se geralmente que, quando um paciente apresenta apenas ansiedade leve sem outras complicações, ele pode ser ajudado ao se estabelecer um relacionamento de confiança com o profissional e também através da comunicação de informações realistas sobre o tratamento odontológico. O vínculo entre o dentista e o paciente pode ser estabelecido a partir de uma relação de compromisso que se estabelece entre as partes, pautada no cumprimento de acordos feitos (como horários de atendimento), na disponibilidade do profissional em sanar dúvidas, explicitar condutas, não produzir sons e barulhos desnecessários, não deixar instrumentos no campo de visão do paciente e avançar o trabalho à medida que o paciente se sente confortável ${ }^{29}$. Essas estratégias de redução da ansiedade relativamente simples podem proporcionar ao paciente uma sensação de controle e de previsibilidade em relação ao tratamento, favorecendo a cooperação e tornando a ida ao dentista menos custosa emocionalmente.

Com pacientes com níveis mais elevados de aversão ao tratamento odontológico pode-se tomar parte de outras abordagens de controle da ansiedade. Tais indivíduos podem precisar de suporte farmacológico específico (como óxido nitroso ou sedação oral com benzodiazepínicos), além do uso de estratégias como distração, relaxamento ou desenvolvimento de estratégias de enfrentamento 7 . Os benzodiazepínicos são os fármacos ansiolíticos e sedativos utilizados com maior frequência na Odontologia e em geral são empregados para sedação pré-operatória logo antes do procedimento e como um auxiliar do sono na noite anterior à cirurgia. Em um paciente ansioso quanto ao procedimento, a ação ansiolítica dos benzodiazepínicos pode produzir redução perceptível na pressão arterial e na frequência cardíaca ${ }^{30}$.

Bernson et al. ${ }^{8}$ conduziram entrevistas com indivíduos diagnosticados com medo de dentista, e quando indagados sobre quais técnicas os pacientes costumavam aplicar para dar continuidade o tratamento, dois dos quatro temas principais que emergiram foram "interação confiável com o dentista" e "buscar estar sob controle da situação". O desenvolvimento de um senso de controle envolve assumir um papel ativo nos procedimentos e se comunicar com o dentista, perguntando, por exemplo, qual procedimento está sendo realizado, quanto tempo irá durar, se irá doer ou não, e outras perguntas que podem aumentar o conhecimento do paciente acerca das condutas que estão sendo tomadas. O tema geral que emergiu dessas entrevistas foi que as pessoas com medo veem o atendimento como um esforço mútuo por parte do paciente e do dentista, e que ambas as partes precisam fazer esforços para que o tratamento prossiga sem problemas. Na sequência, são descritos métodos de manejo do paciente para que o CD e os pacientes possam usar tanto como uma prevenção de respostas de ansiedade como também na presença destas, visando facilitar o processo de tratamento odontológico.

\section{Falar-mostrar-fazer}

Embora a técnica de Falar-Mostrar-Fazer tenha sido desenvolvida originalmente para uso na odontopediatria, ela também pode ser aplicada a adultos com ansiedade, promovendo uma sensação de controle e previsibilidade. Uma variação supostamente usada com adultos é o "Explicar-Perguntar-Mostrar-Fazer", a qual visa estabelecer uma situação de cooperação mútua. Nessa variação, sendo que cada estágio envolve explicar o que o dentista gostaria que acontecesse ou sugerir um próximo passo no atendimento ao paciente, respondendo a todas as perguntas que ele (paciente) possa ter e depois que o paciente receber todas as informações necessárias, solicitar permissão para prosseguir para os estágios "mostrar" e "fazer". Tal processo busca considerar os medos e fobias do paciente, respeitando-o e avançando no procedimento conforme o paciente vai permitindo. No entanto, não há pesquisas conhecidas sobre a eficácia dessa abordagem . 


\section{Distração}

Armfield e Heaton ${ }^{7}$ relatam que a técnica de distração, sendo esta direcionar a atenção do paciente para estímulos visuais ou auditivos alternativos específicos no consultório odontológico, pode ser benéfica para pacientes com ansiedade dental leve a moderada. Estudos mostram que óculos de realidade virtual são capazes de reduzir a pressão arterial e a pulsação e aumentar o limiar de dor em pacientes submetidos a procedimentos odontológicos mais invasivos ${ }^{7}$.

Embora várias opções estejam disponíveis para o clínico, desde música a vídeos, jogos de computador e óculos 3D para assistir a filmes, atualmente existem evidências variadas sobre a eficácia dessas práticas de distração na clínica odontológica. Um estudo de metanálise realizada por Klassen et al. ${ }^{13}$, descobriu, a partir da análise de 19 ensaios clínicos randomizados, que a musicoterapia reduz a dor e a ansiedade de crianças submetidas a procedimentos odontológicos. Já em um estudo recente de Kim et al. ${ }^{12}$, com adultos que foram submetidos à cirurgia de extração dos terceiros molares, foi observado pelos pesquisadores redução na ansiedade para um grupo tratado com música intraoperatória em comparação a um grupo tratado sem música. Os sinais vitais dos pacientes (frequência cardíaca, frequência respiratória e pressão arterial) foram monitorados durante todo o procedimento cirúrgico. A análise unidirecional da covariância, usando a ansiedade perioperatória como covariância, foi utilizada para comparar os níveis de ansiedade intraoperatória e as percepções perioperatórias da dor entre os dois grupos. A análise de variância das medidas repetidas foi utilizada para comparar alterações nos sinais vitais nos estágios cirúrgicos entre os dois grupos.

Os sinais vitais mudaram substancialmente durante a cirurgia, de acordo com o estágio do procedimento. Para ambos os grupos, os sinais aumentaram da linha de base e atingiram valores máximos no momento da incisão inicial, diminuindo rapidamente e atingindo o platô dentro dos limites normais. Não houve diferenças significativas entre os grupos na pressão arterial; no entanto, o grupo tratado com música mostrou uma mudança significativamente menor na frequência cardíaca do que o grupo controle. O grupo tratado com música relatou significativamente menos ansiedade intraoperatória do que o grupo controle sem música ao controlar os níveis de ansiedade pré-operatória.

\section{Reforço positivo}

O estímulo reforçador, para a Análise do Comportamento, é considerado uma consequência do comportamento, o qual mantém ou aumenta a probabilidade de um determinado comportamento voltar a ocorrer em condições semelhantes. Segundo Skinner ${ }^{31}$, um indivíduo tende a se sentir bem consigo mesmo quando sente os efeitos de contingências de reforçamento positivo. Em linhas gerais, o que se define como bem-estar do indivíduo é uma maior probabilidade de agir de modo a produzir consequências positivas e se afastar, pospor ou evitar estímulos aversivos.

Particularmente em relação às crianças, mas também aos adultos, o reforço positivo, pode fornecer um incentivo útil para a cooperação ou outros comportamentos apropriados no contexto durante o atendimento. Por exemplo, ao emitir comportamentos de cooperação, o paciente pode produzir consequências reforçadoras, como ganhar algo de seu interesse (uma escova de dente, por exemplo). Assim, o reforço positivo (escova de dente) aumenta a probabilidade de que o paciente volte a emitir comportamentos de colaboração e também pode possibilitar o estabelecimento de uma relação mais próxima entre o paciente e o CD.

Outra técnica possível é a que envolve a possibilidade do paciente, após emitir comportamentos desejados dentro do contexto odontológico, produzir, como consequência, breves pausas no andamento do procedimento. Nesse caso, os comportamentos de cooperação seriam mantidos por reforçamento negativo, isto é, após sua emissão, a consequência produzida seria o afastamento temporário de estímulos aversivos. Assim, nesta técnica, que 
é muito semelhante à sinalização, breves períodos de "fuga" do tratamento odontológico em andamento são fornecidos à pessoa que depende de um comportamento cooperativo ou apropriado. O reforço verbal positivo e um breve período de fuga (5 a 10 segundos) ocorre quando o paciente permanece deitado, segue as orientações dadas pelo dentista e coopera com este, enquanto o comportamento disruptivo atrasa a fuga até que comportamentos de cooperação sejam novamente emitidos ${ }^{32}$.

\section{Respiração com foco no relaxamento}

Quando as pessoas encontram-se em situações de estresse durante períodos prolongados, raramente conseguem que seus músculos relaxem. Isto provoca mal-estar no indivíduo, apreensão, irritação e cansaço constantes. Além disso, a ansiedade pode dificultar ou inibir muitas formas de comportamento social, pois interagir socialmente (cumprimentar as pessoas, conversar, manter um diálogo, fazer perguntas, e outros comportamentos) passa a ser interpretado como uma tarefa penosa e extremamente complexa, dificultando ainda mais as idas às consultas odontológicas ${ }^{33}$. Há registros na literatura que um exercício benéfico para grande parte de pacientes com medo é o relaxamento através da respiração controlada. As mudanças fisiológicas que acompanham a respiração relaxada formam efetivamente uma contrapartida e, portanto, são incompatíveis com a reação de "luta ou fuga" que caracterizam os indivíduos com ansiedade.

A respiração lenta e constante por 2 a 4 minutos é considerada eficaz na redução da frequência cardíaca de pacientes que se queixam de ansiedade ${ }^{16}$. Ackley ${ }^{6}$, por outro lado, recomendou que os pacientes sejam orientados a respirar tão lentamente, de tal forma que se, hipoteticamente, uma pena estiver embaixo do nariz, ela não se moverá. Essas técnicas de respiração podem ser ensinadas com bastante facilidade na clínica odontológica e praticadas em casa pelo paciente antes de uma consulta.

\section{Reestruturação cognitiva}

Enquanto outras técnicas de manejo do paciente com ansiedade, medo e fobia visam desviar a atenção do paciente da situação que evoca o medo, a reestruturação cognitiva visa, por sua vez, alterar e reestruturar o conteúdo das cognições negativas de uma pessoa, além de aumentar o controle do paciente sobre tais pensamentos. O processo envolve a identificação de interpretações errôneas e pensamentos catastróficos frequentemente associados ao medo odontológico, desafiando as evidências do paciente e substituindo-os por pensamentos mais realistas. Alguns exemplos de pensamentos catastróficos podem ser "Irei morrer com a anestesia", "não vou conseguir suportar a dor", "não vou conseguir me recuperar bem após o procedimento".

Utilizando-se fundamentalmente dos princípios da Terapia Racional-Emotivo-Conductal (TREC), pode-se afirmar que o pensamento irracional é a principal causa de transtorno emocional, visto que, consciente ou inconscientemente, a pessoa pensa de maneira ilógica e pouco realista ${ }^{34}$. Logo, o paciente sente-se ansioso, não frente à situação objetiva, mas à interpretação que o mesmo faz dessa situação.

O principal método de substituição de uma crença irracional por uma racional denomina-se refutação ou debate e é, fundamentalmente, uma adaptação do método científico ao dia a dia, estratégia pela qual questionam-se hipóteses e conceitos para determinar sua legitimidade.

As evidências para a potencial eficácia da reestruturação cognitiva vem principalmente de estudos com pacientes que procuram psicólogos clínicos na abordagem cognitiva. No entanto, algumas evidências apontam que as habilidades necessárias para realizar a reestruturação cognitiva podem estar ao alcance dos profissionais da área odontológica, através de treinamento e supervisão especiais ${ }^{34}$. 


\section{Dessensibilização sistemática}

A dessensibilização sistemática se caracteriza por uma exposição gradual aos estímulos geradores de ansiedade. Para tal exposição, cria-se uma escala hierárquica de ansiedade, identificando o grau de aversão a cada estímulo ${ }^{35}$. No consultório odontológico, tal método de enfrentamento da ansiedade pode ser executado pedindo que o paciente faça uma lista com todos os estímulos relacionados ao contexto odontológico que lhe geram ansiedade. Em seguida, o mesmo irá enumerar progressivamente o estímulo menos ansiogênico até aquele que produz maior ansiedade (hierarquia da ansiedade). A partir dessa escala, o dentista apresentará ao paciente primeiramente o estímulo que produz menor ansiedade. Após esse estímulo não ser mais gerador de medo, e o paciente conseguir lidar com ele de maneira adequada, passa-se ao próximo estímulo da escala. Por exemplo, para um paciente com medo de injeções (nível máximo de ansiedade), o dentista pode primeiro mostrar-lhe a seringa (nível menor de ansiedade) e explicar suas partes e finalidade até que o paciente possa visualizar a seringa com pouca ou nenhuma ansiedade. Em seguida, cirurgião pode colocar a seringa com a agulha tapada na boca do paciente para simular a injeção, mantendo a seringa no lugar durante o tempo de uma injeção típica. Em concomitância, o paciente deve ser incentivado a usar estratégias de relaxamento para gerenciar a ansiedade causada por este exercício, e essa etapa é repetida até que o paciente expresse uma ansiedade tolerável ou nenhuma ansiedade. Por fim, o dentista - com a permissão do paciente - pode prosseguir com a técnica anestésica, replicando o local e o período de tempo demonstrado nas etapas anteriores.

Hakeberg et al. ${ }^{11}$ evidenciaram que pacientes com medo odontológico que concluíram um programa de dessensibilização sistemática mostraram maior redução do medo e melhora no humor após receber tratamento odontológico em comparação com aqueles pacientes pré-medicados com Diazepam antes do tratamento odontológico.

O processo de exposição pode ser sistematizado ainda mais usando a exposição baseada em vídeo com imagens que contenham os objetivos a serem alcançados pelo paciente. Seja via computador ou pessoalmente, a dessensibilização sistemática permite que os pacientes sejam expostos gradualmente a estímulos geradores de ansiedade até que diminua a adversidade a esses estímulos e então a conduta odontológica possa ser realizada.

Entendendo que a Psicologia é uma ciência com múltiplas formas de entendimento da condição humana e distintas abordagens, como a comportamental e a cognitiva, pode-se afirmar que tal multiplicidade reflete em diferentes manejos da ansiedade em pacientes odontológicos. Isto é, não existe apenas uma forma de lidar com a ansiedade e que precisamos levar em consideração as especificidades de cada paciente e também o preparo técnico do dentista em lidar com a ansiedade do paciente.

\section{CONCLUSÃo}

Embora a prática da Odontologia implique no uso de uma variedade de habilidades, a profissão envolve uma interação muito próxima entre o profissional e o paciente. E, embora o paciente tenha a expectativa de que seus problemas de saúde bucal sejam resolvidos, ele também entra em um relacionamento com o profissional da Odontologia com a expectativa de que será tratado de maneira cuidadosa e respeitosa. Para o dentista, há satisfação no trabalho com o manejo bem-sucedido da ansiedade, do medo e da fobia dental do paciente e de sua saúde bucal. E para as pessoas com medo odontológico, dada a forte associação entre ansiedade e visita odontológica, aprender a gerenciar sua ansiedade também ajudará a melhorar sua saúde bucal ${ }^{20}$. 
A abordagem das questões do medo e da ansiedade odontológica deve contribuir para a sua prevenção, diagnóstico e o seu tratamento, o que implicará novas e diferentes abordagens terapêuticas e uma melhor educação em saúde. Nesta circunstância, a influência de uma formação voltada aos aspectos psicológicos do paciente, tornam-se notável, visto que habilita o CD a saber interpretar indícios e reações que revelam temores e fragilidades dos pacientes, com abordagens metodológicas e farmacológicas apropriadas.

Uma possível limitação do presente estudo caracteriza-se pela busca de artigos usando apenas o termo "ansiedade", visto que, caso tivessem sido inseridos os descritores "medo de dentista" ou "fobia de dentista" poderiam ser identificados mais materiais sobre o assunto.

São necessários mais estudos que ponderem a efetividade das estratégias aqui sugeridas, uma vez que é possível que pacientes excessivamente opositores quanto ao tratamento odontológico estejam também sob influência de outras condições não estritamente associadas ao âmbito odontológico. Logo, um trabalho psicológico prévio ou em concomitância ao tratamento odontológico, e que atue na identificação e manejo desses fatores ansiogênicos, pode se mostrar positivo para casos mais intensos, nos quais a admissão de estratégias somente por parte do CD apresentem-se insuficientes.

\section{REFERÊNCIAS}

1. Seger L. Psicologia e odontologia: uma abordagem integradora. 4. ed. São Paulo: Santos; 2002.

2. Castillo ARGL, Recondo R, Asbahr FR, Manfro GG. Transtornos de ansiedade. Rev Bras Psiquiatr 200o Dec;22(2):20-3.

3. American Psychiatry Association. Diagnostic and statistical manual of mental disorders - DSM-5. 5th.ed. Washington: American Psychiatric Association; 2013

4. Mayer TMAS, Assis PD, Nascimento MG, Barreto KA, Silva CMMP, Colares V. Prevalência de ansiedade relacionada ao tratamento odontológico em adolescentes: revisão integrativa da literatura. Arch Health Invest. 2019 Set;8(3):145-9.

5. Carter AE, Carter G, Boschen M, AlShwaimi E, George R. Pathways of fear and anxiety in dentistry: a review. World ] Clin Cases. 2014 Nov 16; 2(11):642-53.

6. Ackley DC. Dental fear: Aren't you tired of it?. Dent Today. 2003 Jan;22 (1):96-102.

7. Armfield JM, Heaton L]. Management of fear and anxiety in the dental clinic: a review. Aust Dent ]. 2013 Dec;58(4):390-531.

8. Bernson JM, Hallberg, LR M, Elfström ML, Hakeberg M. 'Making dental care possible - a mutual affair': a grounded theory relating to adult patients with dental fear and regular dental treatment. Euro ] Oral Sci. 2011 Oct;119(5):373-80

9. Carvalho RWF, Falcão PCCB, Vasconcelos BCE, Pereira MAS, Bastos AS, Campos C]L, et al. Ansiedade frente ao tratamento odontológico: prevalência e fatores preditores em brasileiros. Cienc saude coletiva. 2011 ]un;17(7):1915-22.

10. Dailey YM, Humphris GM, Lennon MA. Reducing patients' state anxiety in general dental practice: a randomized controlled trial. J Dent Res. 2002 May;81(5):319-22

11. Hakeberg M, Berggren U, Carlsson SC. A 10-year follow-up of patients treated for dental fear. Scand] Dent Res. 1990 Feb;98(1):53 9.

12. Kim Y-K, Kim S-M, Myoung H. Musical intervention reduces patients' anxiety in surgical extraction of an impacted mandibular third molar. J Oral Maxillofac Surg. 2011 Apr;69(4):1036-45

13. Klassen JA, Liang Y, Tjosvold L, Klassen TP, Hartling L. Music for pain and anxiety in children undergoing medical procedures: a systematic review of randomized controlled trials. Ambul Pediatr. 2008 Mar;8(2):117-28.

14. Klingberg G, Broberg AG. Dental fear/anxiety and dental behaviour management problems in children and adolescents: a review of prevalence and concomitant psychological factors. Int ] of Paediatr Dent. 2007 Nov;17(6):391-406

15. Lopes PN, Ponciano E, Pereira A, Medeiros JA, Kleinknecht RA. Psicometria da ansiedade dentária: avaliação das características psicométricas de uma versão portuguesa do dental fear survey. Rev Por Estomatol Cir Maxilofac. 2004 Jan;45(3):133-46

16. Milgrom P, Weinstein P. Dental fears in general practice: new guidelines for assessment and treatment. Int Dental J. 1993 Jun;43(3):288-93. 
17. Murrer RD, Francisco SS. Diagnóstico e manejo da ansiedade odontológica pelos cirurgiões-dentistas. Interação Psicol. 2015 Ago;19 (1):37-46.

18. Nathan JE. Behavioral management strategies foryoung pediatric dental patients with disabilities. ASDC] Dent Child. 2001 Mar;68(2):89-101.

19. Pereira VZ, Barreto R de C, Pereira GAS, Cavalcanti HRBB. Avaliação dos níveis de ansiedade em pacientes submetidos ao tratamento odontológico. Rev Bras Ciênc Saúde. 2013 Mai;17(1):55-4.

20. Peronio TN, Silva AHda, Dias SM. O medo frente ao tratamento odontológico no contexto do Sistema Único de Saúde: uma revisão de literatura integrativa. Braz ] Periodontol. 2019 Mar;29(1):37-43.

21. Silva ACM da. Medo e ansiedade dentária: uma realidade. [Dissertação]. Porto (PT): Universidade Fernando Pessoa, Faculdade de Ciências da Saúde; 2012.

22. Skaret E, Raadal M, Berg E, Kvale G. Dental anxiety and dental avoidance among 12 to 18 year olds in Norway. Euro ] Oral Sci. 1999 Nov; 107:422-8.

23. Ulhoa M, Reis Filho NT, Mariano ]. Medo de dentista: uma proposta para redução da ansiedade odontológica. Roplac. 2015 Jul;5(2):35-41.

24. Barlow DH. Anxiety and its disorders: the nature and treatment of anxiety and panic. 2. ed. New York: Guilford press; 2004.

25. Ekman PE, Davidson R]. The nature of emotion: fundamental questions. New York: Oxford University Press; 1994.

26. Moraes ABA. Comportamento e Saúde: explorando alternativas. Santo André: Editora ARBytes; 1999.

27. Moraes ABA, Pessoti I. Psicologia aplicada à odontologia. São Paulo: Sarvier Editora; 1985.

28. Hu LW, Gorenstein C, Fuentes D. Portuguese version of corah's dental anxiety scale: transcultural adaptation and reliability analysis. Depress Anxiety. 2007 Jan;24(7):467-47.

29. Hupp JR, Tucker MR, Ellis E. Cirurgia oral e maxilofacial contemporânea. 6. ed. Rio de Janeiro: Elsevier; 2015

30. Peterson L]. Peterson's principles of oral and maxillofacial surgery. 3. ed. Raleigh: McGraw-Hill Europe; 2012.

31. Skinner BF. Questões recentes na análise comportamental. 3. ed. Campinas: Papirus; 1991.

32. Ingersoll BD, Nash DA, Blount RL, Gamber C. Distraction and contingent reinforcement with pediatric dental patients. ASDC ] of Dent Child. 1984 May;51(3):203-7.

33. Marinho ML, Caballo VE. Psicologia clínica e da saúde. 1. ed. Londrina: Editora UEL; 2001.

34. Pickrell JE, Heima M, Weinstein P, Coolidge T, Coldwell SE, Skaret E, et al. Using memory restructuring strategy to enhance dental behaviour. Int ] Paediatr Dent. 2007 Nov;17(6):439-48.

35. Moreira MB, Medeiros CAde. Princípios básicos de análise do comportamento. 2. ed. Guarulhos: Artmed; 2018. 